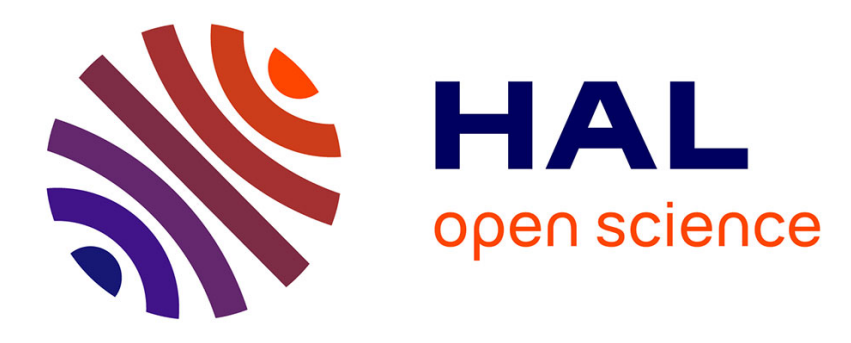

\title{
Light Scattering From Surface Waves On Carbon Dioxide Near Its Critical Point
}

\author{
M. Bouchiat, J. Meunier
}

\section{To cite this version:}

M. Bouchiat, J. Meunier. Light Scattering From Surface Waves On Carbon Dioxide Near Its Critical Point. Journal de Physique Colloques, 1972, 33 (C1), pp.C1-141-C1-148. 10.1051/jphyscol:1972125 . jpa-00214915

\section{HAL Id: jpa-00214915 https://hal.science/jpa-00214915}

Submitted on 1 Jan 1972

HAL is a multi-disciplinary open access archive for the deposit and dissemination of scientific research documents, whether they are published or not. The documents may come from teaching and research institutions in France or abroad, or from public or private research centers.
L'archive ouverte pluridisciplinaire HAL, est destinée au dépôt et à la diffusion de documents scientifiques de niveau recherche, publiés ou non, émanant des établissements d'enseignement et de recherche français ou étrangers, des laboratoires publics ou privés. 


\title{
LIGHT SCATTERING FROM SURFACE WAVES ON CARBON DIOXIDE NEAR ITS CRITICAL POINT
}

\author{
M. A. BOUCHIAT and J. MEUNIER
}

Laboratoire de Spectroscopie Hertzienne, 24, rue Lhomond, Paris $5^{\mathrm{e}}$

\begin{abstract}
Résumé. - Le spectre de la lumière diffusée par les ondes de surface excitées thermiquement sur un interface liquide, a été calculé théoriquement en utilisant la théorie thermodynamique. On a pu prédire des effets caractéristiques d'un processus de dissipation en volume, jusqu'ici non observés ; ces effets devraient surtout se manifester au voisinage des conditions d'amortissement critique, pour lesquelles les ondes de surface cessent de se propager et deviennent exponentiellement amorties. Par contre, dans les conditions de faible et de fort amortissement, on peut identifier le spectre à la puissance spectrale des fluctuations d'un oscillateur harmonique à une dimension.

Nous avons mesuré ce spectre dans le cas de la diffusion par l'interface liquide-vapeur de $\mathrm{CO}_{2}$ près du point critique $\left(T_{\mathrm{c}}-T=0,6^{\circ} \mathrm{C}\right.$, pour des angles de diffusion correspondant à $q \approx 3000 \mathrm{~cm}^{-1}$ ). Le bon accord avec le spectre théorique permet de mettre en évidence de manière significative le processus de dissipation prévu.

Des expériences antérieures réalisées dans la zone de température $30^{\circ} \geqslant T_{\mathrm{c}}-T \geqslant 3,5 \times 10^{-2}{ }^{\circ} \mathrm{C}$ ont été interprétées à l'aide du spectre approché correspondant à celui de l'oscillateur harmonique. La variation de la tension superficielle avec la température a été interprétée à l'aide de la loi empi* rique :

$$
\alpha=\alpha_{0}\left(1-T / T_{\mathrm{c}}\right)^{\mu} \text { avec } \mu=1,253 \pm 0,010 \text { et } \alpha_{0}=77 \pm 5 \text { dynes } / \mathrm{cm} .
$$
\end{abstract}

Il n'est apparu aucune anomalie sur la variation de la viscosité cinétique avec la température.

\begin{abstract}
The spectrum of light scattered from surface waves thermally excited on a liquid interface, has been computed theoretically using the approach of thermodynamic theory. Effects typical of a dissipation process into the bulk (previously unobserved) were predicted ; such effects should show up mainly near critical damping conditions, that is conditions for which surface waves become exponentially damped and no longer propagate. In low and high damping conditions the spectrum can be accurately identified to the fluctuation power spectrum of a one dimensional, harmonic oscillator (H. O. spectrum),

We have measured this spectrum for scattering from the liquid-vapor interface of carbon dioxide near the critical point $\left(T_{\mathrm{c}}-T=0.6^{\circ} \mathrm{C}\right.$ at scattering angles corresponding to $\left.q \approx 3000 \mathrm{~cm}^{-1}\right)$. The agreement with the theoretical spectrum yields a significative evidence for the bulk dissipation process mentioned.

Previous experiments were performed in the temperature range $30^{\circ} \geqslant T_{\mathrm{c}}-T \geqslant 3.5 \times 10^{-2}{ }^{\circ} \mathrm{C}$ and fitted with the help of the approximate $H$. $O$. spectrum. The temperature dependence of the surface tension deduced from the data was fitted by the empirical law
\end{abstract}

$$
\alpha=\alpha_{0}\left(1-T / T_{c}\right)^{\mu} \text { with } \mu=1.253 \pm 0.010 \text { and } \alpha_{0}=77 \pm 5 \mathrm{dynes} / \mathrm{cm} .
$$

No anomaly was noticed in the temperature variation of the shear viscosity.

Thermal motion produces on a liquid interface fluctuations which can propagate as capillary or gravity waves damped by viscosity [1]. These waves scatter a monochromatic incident light beam with a small frequency shift (tens to thousands of hertz) which can be detected by light-beat spectroscopy [2]. This scattering from a liquid surface presents certain analogy with the Brillouin scattering from the bulk : the process can be considered as an inelastic collision between the incident photon and a surface phonon which is either annihilated or created. The first order scattering theory shows that the power spectrum of the electric field scattered at a given angle $\theta$ reflects in the vicinity of the incident light frequency, the power spectrum $P_{q}(\omega)$ of the surface fluctuations having a given wave vector $q$. The relation between $\theta$ and $q$ expresses the conservation of linear momentum in the surface plane, during the scattering process. Investigation of this spectrum can yield the surface tension $\alpha$ and the shear viscosity $\eta$ without disturbing thermal equilibrium. This provides a method quite appropriate to measurements very near the critical point at which the interface disappears: either the liquid-gas interface of a pure fluid near its critical point, or the interface between the two phases of a binary liquid mixture near the critical demixtion point. More conventional techniques perturb the interface and cannot yield the very weak values of $\alpha$ close to $T_{\mathrm{c}}$. The oscillating disk method used to measure the 
shear viscosity of a pure fluid involves corrections near $I ;$ due to the large compressibility of the fluid, which reduce the accuracy of the measurements.

From a practical point of view, surface opalescence plays an important role in the realization of the surface scattering experiment near $T_{\mathrm{c}}$. The surface scattering efficiency is proportional to the light reflectivity and to the mean square of the vertical displacement. As a result of the decrease of the surface tension near $T_{\mathrm{c}}$, the vertical displacement of the interface undergoes increasingly large fluctuations, which contribute to an increase of the scattered intensity. Simultaneously the refraction index difference between the two phases goes to zero, and this tends to reduce the scattering efficiency. Among these two competing effects the second one is the less important, as long as the liquid interface reflects light as a sharp interface. So at certain scattering angles the surface still scatters more light than the bulk. However closer to $T_{c}$ the effective thickness of the interface is not small compared to an optical wavelength. The intensity of the light scattered from the surface decreases drastically while the scattering from the bulk still increases: presently accurate measurements of the spectrum have not yet been performed in such conditions.

In the present paper, we shall first analyse the theoretical problem of the detailed line shape. Then we shall describe our experimental results on the liquid-vapor interface of $\mathrm{CO}_{2}$ near its critical point. Finally we shall show that our surface tension data combined with the results obtained for the compressibility and long range correlation length by other investigators, allow a verification of the scaling law predicted by the Fisk-Widom relation.

A) Thermal fluctuations power spectrum for the vertical displacement of a liquid interface [3], [4]. The model first proposed by Landau and Placzek [5] and reexaminated later by Mountain [6] to describe density fluctuations in the bulk can be adapted to the present problem. In the range of wavelengths and frequencies of interest (a few microns, 10 to $10^{3}$ cycles), one can reasonably describes the evolution of surface fluctuations by using classical hydrodynamic theory. The derivation of the power spectrum then proceeds in two steps :

1. one looks for a solution of the hydrodynamic equations satisfying the boundary conditions at the interface and a set of particular initial conditions;

2. one chooses these initial conditions, using statistical mechanics considerations applied to a system at thermal equilibrium.

We shall present the first derivation of the power spectrum, done in fact in a rather naive and approximate way [3]. We shall show how later we could find unphysical features to the result and how we reformulated the problem to derive a new result in a much more rigorous way [4]. This presentation emphasizes the difference between the old and the new spectrum, which has a fairly simple physical interpretation, we think it is worth to mention. No detailed computation will be given here, we shall rather try to clarify the physical content of the two methods.

I. First Naive Derivation. -- The fluctuation amplitude being small compared to the wavelength the equations of hydrodynamics can be linearized.

The fluid is supposed incompressible. This is justified because surface and sound waves of a given wavelength have very different velocities and are uncoupled even for a pure fluid near its critical point [7].

So we look for solutions of the volume equations, expressing mass and linear momentum conservation, which satisfy the boundary conditions at the interface.

We can treat independently surface deformations having different wave vectors $\mathbf{q}$ : the fluctuations being small have independant time evolution and also translational invariance of the surface properties imposes the absence of correlations between fluctuations of different $\mathbf{q}$ considered at the same time.

In hydrodynamic text books one generally uses to solve the problem by looking for solutions of the following type :

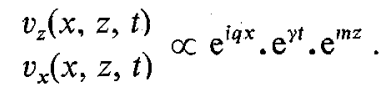

$\mathrm{O} z$ denoting the vertical axis, $z=0$ the plane of the interface, $\mathrm{O} x$ an axis parallel to the wave vector $\mathbf{q}$. The vertical displacement of the interface $\zeta(x, t)$ is related to the velocity $\mathbf{v}$ of the fluid by :

$$
\dot{\zeta}(x, t)=v_{z}(x, z=0, t) .
$$

A solution of the preceding type is physically acceptable only if the two following conditions are fulfilled:

$$
\operatorname{Re}(\gamma)<0
$$

and

$$
z \cdot \operatorname{Re}(m)<0 .
$$

Then it can be shown that for each value of $q$ there are two possible values for $\gamma\left(\gamma_{1}\right.$ and $\left.\gamma_{2}\right)$ which are the roots of the dispersion equation $D(\gamma)=0$. For each value of $\gamma$ the $z$ dependence is completely specified, as the sum of 2 exponential terms corresponding to $m=q$ and $\left(^{1}\right)$ $m=q \sqrt[+]{1+2 \gamma \tau_{0}}$ with a relative weight depending on $\gamma$ only. Finally one can obtain a solution of type (1) which depends on two arbitrary constants only, the weights of the two time exponentials; these constants become completely specified if the vertical displacement of the interface and its time derivative are known at the initial time. Let us write down the complete expression of the solution for $v_{z, q}(z, t)$ and $\zeta_{q}(t)$, the

(1) The upper script + means that one considers the determition of the square root having a positive real part. 
Fourier transforms with respect to $x$ of the vertical velocity and the vertical displacement :

$$
\begin{aligned}
& v_{z, q}(z, t)= \sum_{\substack{\mu, \nu=1,2 \\
\mu \neq \nu}} \frac{\mathrm{e}^{\gamma_{\nu} t}}{\left(\gamma_{\mu}-\gamma_{\nu}\right) \tau_{0}}\left[\zeta_{q}(0) \gamma_{\mu}-\dot{\zeta}_{q}(0)\right] \times \\
& \times\left\{\left(1+\gamma_{\nu} \tau_{0}\right) \mathrm{e}^{q z}-\mathrm{e}^{q \tau \sqrt[+]{1+2 \gamma_{\nu t 0} z}}\right\} \\
& \zeta_{q}(t)=\zeta_{q}(0) \frac{\gamma_{2} \mathrm{e}^{\gamma_{1} t}-\gamma_{1} \mathrm{e}^{\gamma_{2} t}}{\gamma_{2}-\gamma_{1}}+\dot{\zeta}_{q}(0) \frac{\mathrm{e}^{\gamma_{2} t}-\mathrm{e}^{\gamma_{1} t}}{\gamma_{2}-\gamma_{1}}
\end{aligned}
$$

with $\tau_{0}=\rho / 2 \eta q^{2}$.

It will be useful for the following to write the last expression more simply :

$$
\zeta_{q}(t)=\zeta_{q}(0) f(t)+\dot{\zeta}_{q}(0) g(t) .
$$

For a simple liquid

$$
D(\gamma)=y+\left(1+\gamma \tau_{0}\right)^{2}-\sqrt[+]{1+2 \gamma \tau_{0}}
$$

with

$$
y=\frac{\alpha \rho}{4 \eta^{2} q} .
$$

The dispersion equation has always 2 roots, real or complex conjugate.

From these results one can note that the method used does not correspond to the search of the $z$ Laplace$t$ Fourier-transform of the most general solution, since in the expression (1) we cannot exclude complex values of $\gamma$ and $m$. Consequently we cannot prove that the solution obtained with this particular set of initial conditions is unique. However it is the solution we have used in our first derivation of the fluctuation power spectrum [3]. With this solution we are in a position to perform the second step : the choice of the initial conditions $\zeta_{q}(0), \dot{\zeta}_{q}(0)$. Let us consider an ensemble of systems representative of thermal equilibrium. We devide it in several classes, putting all the systems having the same fluctuations $\left(\zeta_{q}(0), \dot{\zeta}_{q}(0)\right)$ at a given time $t=0$, in the same class. Let us first look at a single class. Following Landau, we assume that the hydrodynamic solution $\zeta_{q}(t)$ (eq. (2c)) represents the averaged value of $\zeta_{q}(t)$ for the systems of this particular class :

$$
\begin{array}{r}
\zeta_{q}(0) \zeta_{q}^{*}(t)=\left|\zeta_{q}(0)\right|^{2} f_{q}(t)+\zeta_{q}(0) \dot{\zeta}_{q}^{*}(0) g_{q}(t) \\
\text { for } t>0 .
\end{array}
$$

Then we consider all the classes. From one to another $\zeta_{q}(0)$ and $\dot{\zeta}_{q}(0)$ vary at random but $f(t)$ and $g(t)$ stay the same. Performing the average on the whole ensemble, we can write :

$$
\begin{array}{r}
\overline{\zeta_{q}(0) \zeta_{q}^{*}(t)}=\overline{\left|\zeta_{q}(0)\right|^{2}} f_{q}^{*}(t)+\overline{\zeta_{q}(0) \dot{\zeta}_{q}^{*}(0)} g_{q}^{*}(t) \\
\text { for } t>0 .
\end{array}
$$

The corresponding expression for $t<0$ is simply obtained by noting that the correlation function $\overline{\zeta_{q}(0) \zeta_{q}^{*}(t)}$ is an even function of $t$ as a result of the stationnarity of the random process. We thus obtain for $t>0$ or $t<0$ :

$$
\overline{\zeta_{q}(0) \zeta_{q}^{*}(t)}=\overline{\left|\zeta_{q}(0)\right|^{2}} f_{q}^{*}(|t|)+\overline{\zeta_{q}(0) \dot{\zeta}_{q}^{*}(0)} g_{q}^{*}(|t|) \text {. }
$$

The averaged quantities in the above equation can be evaluated by applying statistical mechanics at thermal equilibrium :

$$
\begin{aligned}
\overline{\zeta_{q}(0) \dot{\zeta}_{q}^{*}(0)} & =0 \\
\overline{\left.\zeta_{q}(0)\right|^{2}} & =\frac{k_{\mathrm{B}} T}{\alpha q^{2}} .
\end{aligned}
$$

The first relation will be justified later on; the second can be derived by evaluating the entropy variation associated to the production of the fluctuation $\zeta_{\mathrm{q}}$.

Finally the correlation function, or its time-Fouriertransform i. e. the power spectrum of the fluctuations $P_{q}(\omega)$, can be directly deduced from $f_{q}(t)$, that is from the solution of the hydrodynamic problem corresponding to the initial conditions $\zeta_{q}(0)$ given, and $\dot{\zeta}_{q}(0)=0$. The results are the following:

correlation function,

$$
G_{q}(t)=\frac{k_{\mathrm{B}} T}{\alpha q^{2}} \frac{\gamma_{2} \mathrm{e}^{\gamma_{1}|t|}-\gamma_{1} \mathrm{e}^{\gamma_{2}|t|}}{\gamma_{2}-\gamma_{1}}
$$

power spectrum,

$P_{q}(\omega)=\frac{k_{\mathrm{B}} T}{\pi \alpha q^{2}} \frac{\gamma_{2} \gamma_{1}\left(\gamma_{2}+\gamma_{1}\right)}{\left(\omega^{2}-\gamma_{1} \gamma_{2}\right)^{2}+\omega^{2}\left(\gamma_{1}+\gamma_{2}\right)^{2}}$.

This spectrum is very well known from people who use to analyse Brillouin scattering. As noticed by Litovitz and al. [8] it is exactly similar to the spectrum of a. resonant system driven by thermal noise ; in the following we shall denote it as the $\mathrm{H}$. O. spectrum, meaning so that this is the fluctuations power spectrum of a onedimensional harmonic oscillator thermally excited.

If the two roots $\gamma_{1}$ and $\gamma_{2}$ of the dispersion equation: are complex conjugate i. e. $\gamma_{2}^{1}= \pm i \omega_{q}-1 / \tau_{q}$, our result means that the thermal excitation of the surface waves is completely analog to that of a gas of free surface phonons having frequency $\omega_{q} / 2 \pi$ and life time $\tau_{q}$.

II. CritrcisM. - We have obtained the correlation function of the fluctuations from considerations of equilibrium statistical mechanics relative to the liquid surface alone. This may appear rather surprising since the surface waves have a penetration depth of the order of $1 / q$ and a displacement at the surface cannot be dissociated from a liquid flow underneath, and vice versa. Looking more insight we found that the preceding derivation implies some consequences which are 
unreasonable and cannot be accepted. Namely, providing $\zeta_{q}(0)$ and $\dot{\zeta}_{q}(0)$ are given the hydrodynamic solution used above specifies completely the state of the whole fluid at $t=0$ : see for instance the expression of $v_{z, q}(z, t)$ given by eq. $(2 a) ; v_{x q}(z, t)$ is quite similar. In particular one can compute the expression $v_{z q}(z, 0)$ $\zeta_{q}^{*}(0)$ in terms of $\zeta_{q}(0)$ and $\dot{\zeta}_{q}(0)$, and its statistical average using the statistical averaged values of $\overline{\left|\zeta_{q}(0)\right|^{2}}$ and $\overline{\zeta_{q}(0) \dot{\zeta}_{q}^{*}(0)}$; one finds that $\overline{v_{z q}(z, 0) \zeta_{q}^{*}(0)}$ is not identically 0 for $z \neq 0$ and that it depends on the damping conditions. So, implicitly, we have done an assumption about the correlation function between the vertical displacement of the interface and the vertical component of the liquid velocity underneath, considered at the same time. This assumption is not physically acceptable. We must have in fact :

$$
\overline{v_{z q}(z, 0) \zeta_{q}^{*}(0)}=0
$$

not only for $z=0$, as we have supposed by putting $\overline{\dot{\zeta}_{q}(0) \zeta_{q}^{*}(0)}=0$, but also anywhere inside the fluid. This statement can be proved to be correct by noting that the quantity to be averaged is an impair function of time, and that the hamiltonian of the microscopic system is time reversal invariant. A detailed proof is given in the appendix. Note also that it is physically unreasonable to find equilibrium statistical properties of a system which depend on its dissipation properties ; $\overline{v_{z q}(z, 0) \zeta_{q}^{*}(0)}$ has to be independant of the damping conditions. As a conclusion the problem has to be reformulated.

III. A MORE CORRECT DERIVATION OF THE SPECTRUM [4]. - Turning back to the hydrodynamic problem we found that the method used at first was not quite correct, because the influence of the initial conditions on the form of the solution was not correctly treated. The most general solution of the hydrodynamic problem [4] is less simple than assumed by eq. (1) : it is not the product of a function of $t$ by a function of $z$. The previous assumption of an exponential decay for $v$ was overly restrictive. One finds there is an infinity of solutions corresponding to a particular set $\zeta_{q}(0), \dot{\zeta}_{q}(0)$ of initial conditions (with a single one having the exponential behaviour). To specify completely the solution it is necessary to give something more at $t=0$, for instance $v_{z q}(z, 0)$ for all $z$. In particular we have derived (through its Laplace transform) the unique solution corresponding to $v_{z q}(z, 0) \equiv 0$ for all $z$, and to $\zeta_{q}(0)$ given. From this solution it is very easy to derive the power spectrum of the surface fluctuations [4], taking now into account that:

$$
\begin{gathered}
\overline{v_{z q}(z, 0)} \zeta_{q}^{*}(0)=0 \text { for any } z \\
\overline{\left|\zeta_{q}(0)\right|^{2}}=\frac{k_{\mathrm{B}} T}{\alpha q^{2}}
\end{gathered}
$$

at thermal equilibrium. The results for the free interface of a pure liquid are the following:

$$
\begin{aligned}
\tilde{\zeta}_{q}(s)= & \int_{0}^{\infty} \zeta_{q}(t) \mathrm{e}^{-s t} \mathrm{~d} t \\
= & \zeta_{q}(0)\left\{\frac{(1+S)^{2}-\sqrt[+t]{1+2 S}}{s\left[y+(1+S)^{2}-\sqrt[+]{1+2 S}\right]}\right\}_{s=s \rho / 2 n q^{2}} \\
P_{q}(\omega) & =\frac{1}{\pi} \operatorname{Re}\left\{\zeta_{q}(s) \zeta_{q}^{*}(0)\right\}_{s=-i \omega} \\
& =-\frac{q}{\rho} \tau_{0}^{2} \operatorname{Im}\left\{\frac{1}{D\left(i \omega \tau_{0}\right)}\right\} \frac{k_{\mathrm{B}} T}{\pi \omega}
\end{aligned}
$$

$D(S)=0$ represents the dispersion equation (see eq. (3)) .

It is interesting to note that this result can be obtained in a different way, namely by applying the fluctuation-dissipation theorem. This means the following : if one applies on the liquid surface a pressure force which is periodic in space and time, $P_{q}(\omega) \mathrm{e}^{i q x} \mathrm{e}^{i \omega t}$, a deformation $\zeta_{q}(\omega)$ of the surface takes place, which can be written, in a linear approximation, as :

$$
\tilde{\zeta}_{q}(\omega)=\tilde{\chi}_{q}(\omega) P_{q}(\omega) .
$$

The response function $\tilde{\chi}_{q}(\omega)$ can be deduced from the hydrodynamic theory. The corresponding change in energy is $-\tilde{\zeta}_{q}(\omega) P_{q}(\omega)$. Then, in the classical limit $\hbar \omega \ll k T$, the fluctuation-dissipation theorem states that the thermal fiuctuation power-spectrum $\overline{\left.\zeta_{q}(\omega)\right|^{2}}$ is related to the response function by the simple relation :

$$
P_{q}(\omega)=\frac{k_{\mathrm{B}} T}{\pi \omega} \operatorname{Im} \tilde{\chi}_{q}(\omega)
$$

We have computed from hydrodynamics :

$$
\tilde{\chi}_{q}(\omega)=\frac{-\tau_{0}^{2} q / \rho}{y+\left(1-i \omega \tau_{0}\right)^{2}-\sqrt[+]{1-2 i \omega \tau_{0}}}
$$

and verified the relation (12) by combining eq. (10b) and (13).

At this point, it is interesting to discuss some of the properties of the response function given above. If $\tilde{\chi}_{q}(\omega)$ is considered as an analytic function of the complex variable $\omega$, it is found that all its singularities lie in the same half complex plane $(\operatorname{Im} \omega<0)$ as imposed by the causality principle. Among these singularities there are 2 poles which are the roots of the dispersion equation and the characteristic frequencies of the surface waves, but there is also one branch point. This last singularity is quite unusual for a physical system and it makes the difference between a real physical interface and a resonant system.

IV. DIFFERENCE BETWEEN THE TWO SPECTRA AND PHYSICAL INTERPRETATION. -- We have compared numerically the H. O. spectrum $(8 b)$ and the exact 
spectrum $(10 b)$. We have found that the difference is very small and probably undetectable in the case of very low $(y \gg 1)$ or very high damping $(y \ll 1)$ conditions. But there is an intermediate range of damping $(y \sim 1)$ where the difference is unnegligible. This is illustrated on figure 1 . We would like to give a physical interpretation of this result which is related with the very peculiar kind of singularity found for the response function of a liquid surface.

Let us suppose that the liquid previously at rest, undergoes at time $t=0$ some vertical displacement

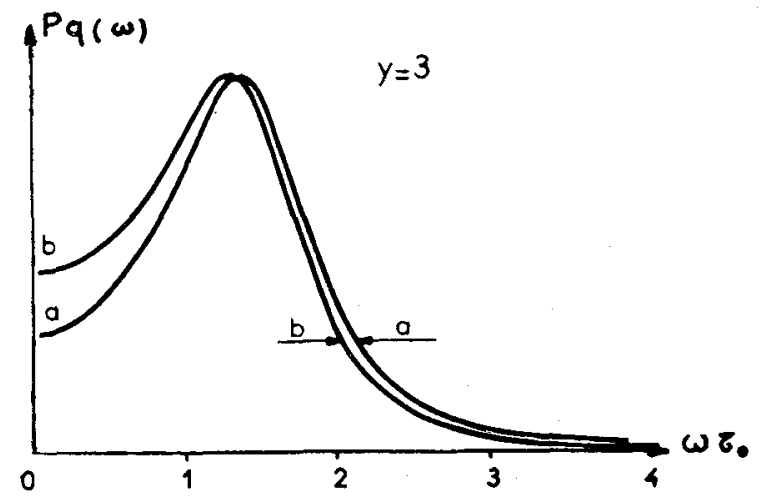

(a)

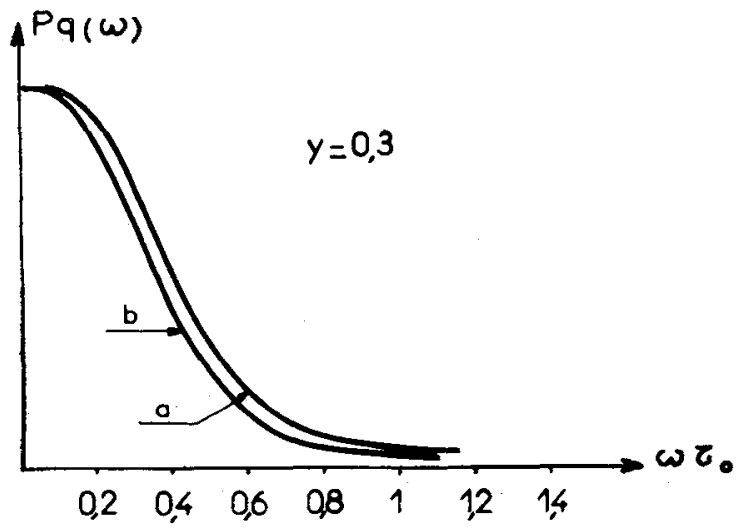

(b)

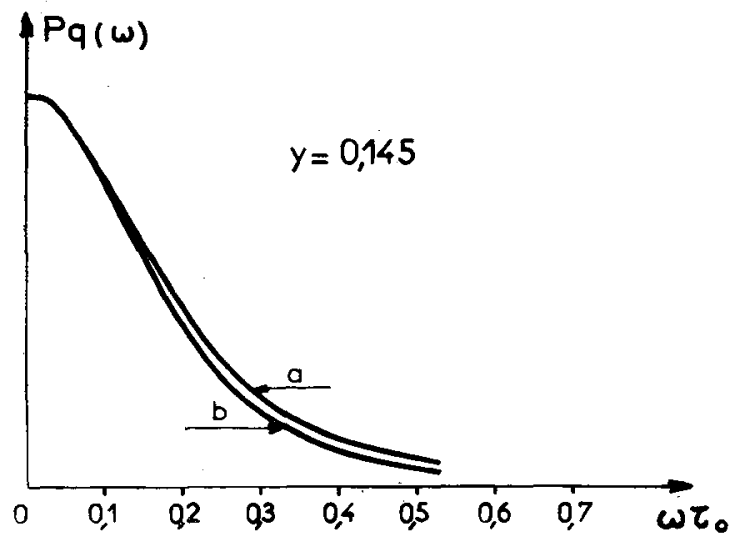

(c)

FIG. 1. - Theoretical power spectrum for the fluctuations of the "vertical displacement of a liquid free surface (curve $a$, eq. $(10 b)$ ), and H. O. spectrum (curve $b$, eq. $(8 b)$ ) corresponding to the same value of the parameter $y=\alpha \rho / 4 \eta^{2} q$. Reduced pulsation units, $\tau_{0}=\rho / 2 \eta q^{2}$. under the action of an external force periodic in space. The displacement produced inside the liquid at the depth $z$ at the time $t^{\prime}>0$ can be expressed as :

$$
u_{q}\left(z, t^{\prime}\right)=\int_{0}^{t^{\prime}} K\left(z, t^{\prime}-t\right) \zeta_{q}(t) \mathrm{d} t
$$

or performing Fourier-transformation with respect to time :

$$
\tilde{u}_{q}(z, \omega)=\tilde{K}(z, \omega) \tilde{\zeta}_{q}(\omega) .
$$

Using hydrodynamics the response function $\widetilde{K}(z, \omega)$ is computed to be :

$$
\widetilde{K}(z, \omega)=\mathrm{e}^{q z}+\frac{i}{\omega \tau_{0}}\left\{\mathrm{e}^{q z} \sqrt[+]{1-2 i \omega t_{0}}-\mathrm{e}^{q z}\right\} .
$$

If $\widetilde{K}(z, \omega)$ would not depend on $\omega, K\left(z, t^{\prime}-t\right)$ would be proportional to $\delta(t)$ and the displacement inside the fluid whatever the depth, would respond instanta neously to the surface excitation. However we can see on the above equation that this is generally not the case. $\widetilde{K}(z, \omega)$ has been written as the sum of two terms, only the first one being frequency independant. It corresponds to the excitation of a potential flow induced by a pressure variation : as the liquid is supposed incompressible, variations of pressure are transmitted instantaneously, producing a displacement at the cote $z$ simultaneously to the surface displacement. The second term in the right hand side of eq. (15), which is frequency dependent, corresponds to the excitation of the rotational flow: in a viscous liquid the surface displacement creates horizontal vortex lines perpendicular to $q$, which diffuse inside the liquid with a time constant $\tau_{0}=\rho / 2 \eta q^{2}$, and progressively excite a retarded displacement under the surface. Let us call $\left[\omega_{1}, \omega_{2}\right]$ the domain of $\omega$ where $P_{q}(\omega)$ takes appreciable values. In low and high damping conditions $\widetilde{K}(z, \omega)$ can be simplified within the range $\left[\omega_{1}, \omega_{2}\right]$; we obtain, for low damping $\left(\omega_{1}, \omega_{2} \gg 1 / \tau_{0} ;\right.$ Fig. $\left.2 a\right)$

$$
\tilde{K}(z, \omega) \approx \mathrm{e}^{q z}
$$

for high damping $\left(\omega_{1}, \omega_{2} \ll 1 / \tau_{0} ;\right.$ Fig. $\left.2 b\right)$

$$
\tilde{K}(z, \omega) \approx \mathrm{e}^{q z}(1+q z) .
$$

In both cases we note that $\widetilde{K}$ is frequency independant in the range $\left[\omega_{1}, \omega_{2}\right]:$ for low damping the rotational flow has not the time to set in before the surface wave has completely decayed ; for high damping the rotational flow sets in very fast, much faster than the wave decay. In both cases the surface waves are not perturbed by the dispersion process associated to the transmission of a motion from the surface to the bulk: the surface response is analog to that of a resonant system. Only in the intermediate case $\omega_{1} \lesssim 1 / \tau_{0} \lesssim \omega_{2}$ (Fig. $2 c$ ), the wave decay and the transmission of motion under the surface take place simultaneously, so the corresponding bulk dissipation process shows off on the spectrum. Note that the motion underneath the surface 


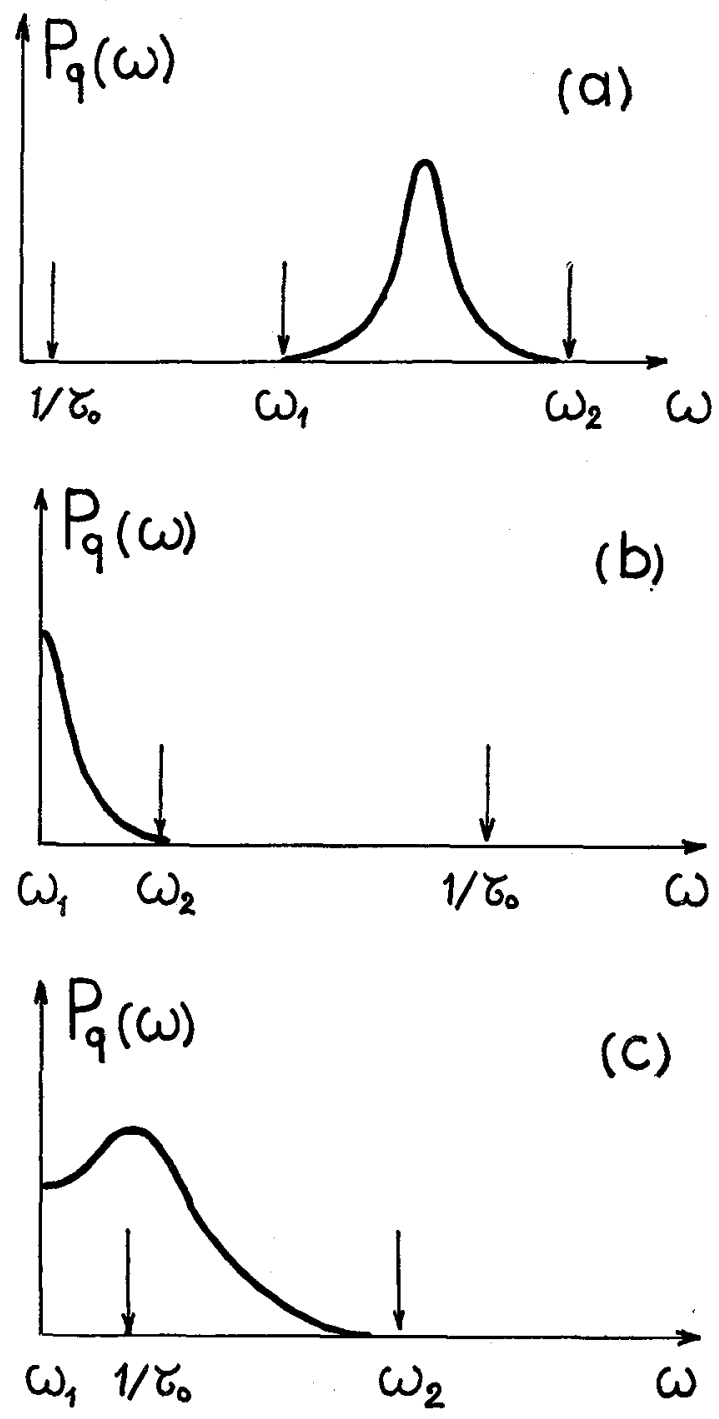

FIG. 2. - Comparison between $1 / \tau_{0}$ and the frequency range characterizing the surface fluctuation power-spectrum, a) low damping case $b$ ) high damping case, $c$ ) intermediate case near critical damping.

does not set in exponentially in time, and $\tau_{0}$ cannot be interpreted as the single relaxation time of the dissipation process; it is more exact to say that there is a continuous distribution of relaxation times, those of the order of $\tau_{0}$ playing the most important role.

As a conclusion a dissipation process in the liquid under the surface is always present but if modifies the spectrum only when its characteristic time is of the order of the time decay of the surface waves. This occurs near critical damping, i. e. near the transition from underdamped to overdamped surface waves $(y \approx 0.145)$.

The reason to mention these effects here is that they play a fairly important role in the study of surface waves near the critical point of a pure fluid. Indeed, the restoring force proportional to the surface tension decreases near $T_{\mathrm{c}}$ as $\left(T_{\mathrm{c}}-T\right)^{1.25}$, while the viscosity does not change markedly, so the waves become more and more damped as one approaches $T_{\mathrm{c}}$; around $T_{\mathrm{c}}-T \sim 10^{-1}{ }^{\circ} \mathrm{C}$ the difference between the $\mathrm{H}$. $\mathrm{O}$. spectrum and the exact spectrum should be clearly detectable. Of course it is then important to use the exact spectral line shape to make accurate determinations of $\alpha$ and $\eta$. As the interface of two different liquids one has to take into account the two different dissipation processes taking place in the liquid layers above and below the surface, with their own characteristic time delay $\tau_{0}^{\prime}=\rho^{\prime} / 2 \eta^{\prime} q^{2}$ and $\tau_{0}=\rho / 2 \eta q^{2}$. As expected the spectrum simplifies greatly if $\tau_{0}^{\prime}=\tau_{0}$, or $\rho^{\prime} / \eta^{\prime}=\rho / \eta$. In the case of $\mathrm{CO}_{2}$, measurements of $\eta$ by a conventional technique [9] indicate that this is a very reasonable approximation. to start with.

B) Experimental results on the liquid-gas interface of $\mathrm{CO}_{2}$. - Let us first indicate that we have been able to test the spectral line shape in the more simple case of the free surface of a pure liquid in the intermediate damping range [10]. The best fit obtained with the approximate $H$. $O$. spectrum or with the exact spectrum were compared; it appeared very clearly that the second was the best. In this experiment the instrumental linewidth is completely negligible and the detection is purely heterodyne. The only two adjustable parameters are $\alpha$ and $\eta$. The best fits with the two different line shapes lead to differences on $\alpha$ and $\eta$ of 6 and 8 per cent.

Figure 3 shows an experimental spectrum resulting from the frequency analysis of light scattered by the liquid-vapor interface of $\mathrm{CO}_{2}$ at $0.59^{\circ} \mathrm{C}$ below $T_{\mathrm{c}}$, for a fairly large scattering angle corresponding to $q=3325 \mathrm{~cm}^{-1}$. We have again represented two theoretical curves corresponding to the best fit obtained using either the approximate $\mathrm{H}$. O. spectrum or the exact spectrum ; but now there is one more adjustable parameter because an important part of the signal comes from self-beating of the scattered light. However, the difference between the experimental and $\mathrm{H}$. O.



FIG. 3. - Frequency analysis of the light scattered from the liquid-vapor interface of $\mathrm{CO}_{2}$ at $T_{\mathrm{c}}-T=0.59^{\circ} \mathrm{C}$ and for $q=3325 \mathrm{~cm}^{-1}$. The points are experimental and the two curves theoretical. The solid line represents the best fit obtained with the correct spectrum (eq. (10b)) and the dashed line the best fit with a H. O. spectrum (eq. ( $8 b)$ ), from the first one we deduce $\alpha=3.321 \times 10^{-2} \mathrm{dyne} / \mathrm{cm}$ and $\eta=0.37 \times 10^{-3}$ poise. 
spectra is significative and illustrates how the dissipation process taking place below and above the interface can modify the fluctuation-spectrum.

Let us now summarize the experimental results obtained by varying the wave-vector and the temperature.

A first set of experiments has been performed with a nearly normal incident light beam, the signal over noise ratio was not then as good as for the spectrum shown on figure 3 and we could not detect any difference between the experimental spectral and the $\mathrm{H}$. O. spectrum. Besides we had not completed yet the exact hydrodynamic derivation. So all the experimental curves of this first set were fitted with a $\mathrm{H}$. O. spectrum. It seems likely that in the range of wave vectors and temperatures under study it affects very little the results. In the temperature range $30 \geqslant T_{\mathrm{c}}-T \geqslant 0.04^{\circ} \mathrm{C}$ (or $10^{-1} \geqslant \varepsilon \geqslant 10^{-4}$ with $\left.\varepsilon=\left(T_{\mathrm{c}}-T\right) / T_{\mathrm{c}}\right)$ the experimental values of the surface tension could be fitted to the power law :

$$
\alpha=\alpha_{0} \varepsilon^{\mu}
$$

with

$$
\begin{aligned}
\alpha_{0} & =77 \pm 5 \text { dynes } / \mathrm{cm}(*) \\
\mu & =1.253 \pm 0.010
\end{aligned}
$$

and for $T_{\mathrm{c}}-T=0.034^{\circ} \mathrm{C}$ assuming that

$$
\frac{\eta}{\rho}=\frac{\eta^{\prime}}{\rho^{\prime}}=\frac{\eta_{\mathrm{c}}}{\rho_{\mathrm{c}}}
$$

we obtained

$$
\eta_{\mathrm{c}}=0.35 \pm 0.05 \times 10^{-3} \text { poise. }
$$

No anomaly was noticed in the temperature variation of $\eta$.

Figure 4 illustrates the results obtained for $\alpha$, and the fit of the power law with the experimental data. In addition near $T_{\mathrm{c}}$ gravity forces begin to play a role in the dispersion law, the capillary and gravity restoring forces, proportional to $\alpha$ and $\Delta \rho g / q^{2}$ may become of the same order of magnitude because the difference between the specific masses of the liquid and gas phases, $\Delta \rho$, decreases less rapidly than $\alpha$. In the range $0.1^{\circ} \geqslant T_{\mathrm{c}}-T \geqslant 0.025^{\circ} \mathrm{C}, \Delta \rho$ can also be obtained from analysis of the spectrum at different $q$. The results shown on figure 4 fit in nicely with more accurate experimental values measured by other investigators, represented by a straight line [11].

A new set of experiments is now under way, using a completely different experimental arrangement: the light beam propagates inside the liquid and the angle of incidence corresponds to total reflection. The scattering efficiency is thus increased specially near $T_{\mathrm{c}}$ : for instance at $T_{\mathrm{c}}-T=10^{-2}{ }^{\circ} \mathrm{C}$ the gain is in a factor 100 . Then the signal over noise ratio is much

$\left.{ }^{*}\right)$ The value of $\alpha_{0}$ given here is different from the one published in reference [7] although it is deduced from the same measurements, because we have corrected a numerical error previously done in the extraction of $\alpha_{0}$ from our data.

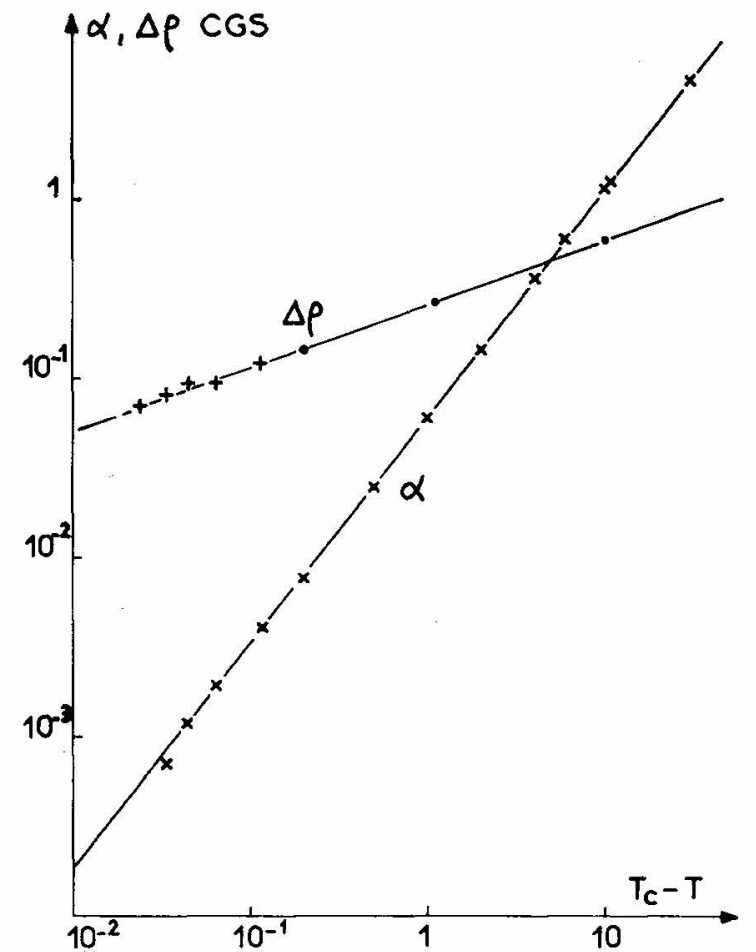

Fig. 4. - The surface teasion $\alpha$ vs $T_{\mathfrak{c}}-T$ using logarithmic scales (the slope of the straight line drawn is 1.25 ) and the difference between the specific masses of the liquid and the vapor $\Delta \rho$ vs $T_{\mathrm{c}}-T$ (the straight line drawn is that of ref. [11]).

better and we can fit the experimental curves with the exact spectrum, as shown on figure 3 . In addition the domain of wave vectors which can be investigated is much vider $\left(200-8000 \mathrm{~cm}^{-1}\right)$, and thus the wavevector definition is substantially increased. We expect that these improvements will allow to approach $T_{\mathrm{c}}$ more closely and to perform more accurate measurements of the viscosity.

The new experimental set-up is schematized on figure 5 . The light source is a $.1 \mathrm{~mW}$ He-Ne laser. The thermostat controls the temperature of the $\mathrm{CO}_{2}$ cell within $2 \times 10^{-4}{ }^{\circ} \mathrm{C}$, during the time of an experiment. The wave vector of the observed fluctuations is fixed by the position of the diaphragm $\mathrm{D}_{2}$ which can be moved along an horizontal axis normal to the reflected light beam. The wave vector and the scattering angle $\Phi$

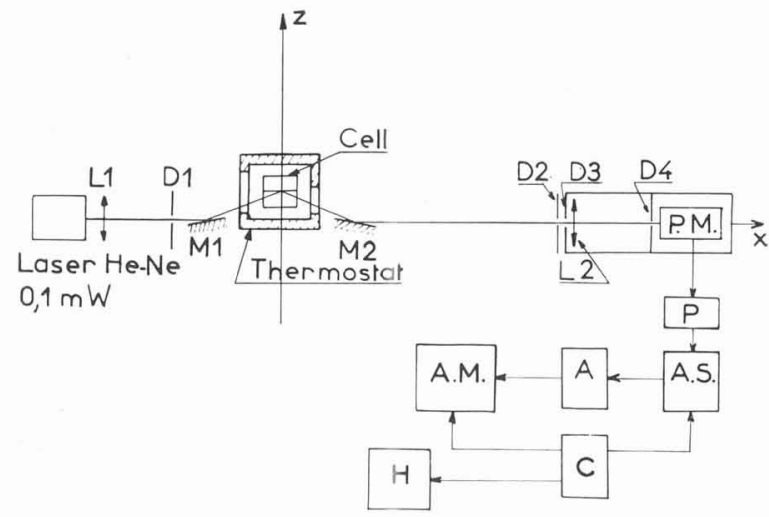

Fig. 5. - Scheme of the new experimental arrangement. 
are connected by the simple relation $q=(2 \pi / \lambda) \Phi$, which stays valid even close to $T_{\mathrm{c}}$ when density gradients become large.

C) Verification of the Fisk-Widom scaling law. We have used the value of the critical exponent $\mu$, which describes the temperature dependence of the surface tension as $T$ approches $T_{\mathrm{c}}$ to examine the validity of the Fisk-Widom scaling law

$$
\mu+v-\gamma^{\prime}-2 \beta=0
$$

$\left(\nu, \gamma^{\prime}\right.$ and $\beta$ being the critical exponents characterizing the temperature variation of the long range correlation length $\xi$, of the isothermal compressibility $K_{T}$, and of $\Delta \rho$ ). Using the following values : $\mu=1.253 \pm 0.01$, $v=0.62 \pm 0.02$ [12]; $\gamma^{\prime}=\gamma=1.17 \pm 0.02$ [13]; $\beta=0.345 \pm 0.01[11]$ we find

$$
\mu+y-\gamma^{\prime}-2 \beta=0.013 \pm 0.03\left({ }^{2}\right) .
$$

The validity of the Fisk-Widom relation itself [14] which connects the magnitude of the four parameters $\alpha$, $\xi, K_{T}$ and $\Delta \rho$, appears to be now an open question which the experiments in progress in different laboratories should help to solve in the near future.

Appendix. - Our purpose here is to show why in a system at thermal equilibrium there is no correlation between the vertical displacement of the interface and the velocity of the fluid below this interface, considered at the same time; i. e.

$$
\bar{v}_{z} \overline{(x, z, t) \overline{\zeta\left(x^{\prime}, t\right)}}=0 .
$$

In this expression the average is taken over an ensemble of systems representative of thermal equilibrium.

For each individual system let us express the quan-

(2) Recent measurements [15] on $\mathrm{CO}_{2}$ above $T_{\mathrm{e}}$ give $v=0.633 \pm 0.01$ and $\gamma=1.219 \pm 0.01$, assuming $\gamma^{\prime}=\gamma$ we find :

$$
\mu+v-\gamma^{\prime}-2 \beta=-0.023 \pm 0.02 \text {. }
$$

tity $v_{z}(x, z) \zeta\left(x^{\prime}\right)$ as a function of the position $q_{i}$ and momentum $p_{i}$ of individual particles :

$$
v_{z}(x, z) \zeta\left(x^{\prime}\right)=\sum_{i} \frac{1}{N} \frac{p_{z, i}}{m_{i}} \cdot \sum_{j} \frac{1}{N^{\prime}} q_{z, j}=A\left(p_{i}, q_{j}\right)
$$

$N$ and $N^{\prime}$ represent the numbers of particles inside small volume elements $V$ and $V^{\prime}$ surrounding the points $x, z$ and $x^{\prime}, z^{\prime}=0$. If $N$ and $N^{\prime}$ are large and if the volume elements have dimensions small compared to $q^{-1}$ the final result will be independant of their choice. Then, the averaged quantity to be computed can be formally written as :

$$
\begin{aligned}
& \overline{v_{z}(x, z) \zeta\left(x^{\prime}\right)}= \\
& =\bar{A} \propto \int_{-\infty}^{+\infty} \mathrm{d} p_{i} \ldots \int_{V^{\prime}} \mathrm{d} q_{j} \ldots A\left(p_{i}, q_{j}\right) \mathrm{e}^{-\beta \operatorname{He}\left(p_{i}, q_{j}\right)}
\end{aligned}
$$

$\mathcal{H}$ is the hamiltonian of the microscopic system; in the absence of a magnetic field it is time reversal invariant. When time is reversed the position of the particles are unchanged and their momentum is reversed: $\mathscr{H}\left(p_{i}, q_{j}\right)=\mathscr{H}\left(-p_{i}, q_{j}\right)$. Besides using the definition of $A$ we can write :

$$
A\left(p_{i}, q_{j}\right)=-A\left(-p_{i}, q_{j}\right)
$$

thus we obtain :

$$
\bar{A}=-\bar{A} \text { or } \bar{A}=0 .
$$

As the result holds for any $x$ and $x^{\prime}$, we also conclude: that :

$$
\int \mathrm{d} x_{0} \overline{v_{z}\left(x_{0}, z\right) \zeta\left(x-x_{0}\right)}=0
$$

or taking the Fourier transform with respect to $x$ :

$$
\overline{v_{z, q}(z) \cdot \zeta_{q}^{*}}=0
$$

Clearly a similar proof could be used to show the absence of correlation between two different physical quantities considered at the same time, providing their product is an odd function of time.

\section{References}

[1] FABELINSKII (I. L.), Molecular Scattering of Light, Plenum Press, N. Y., 1968.

[2] Bouchiat (M. A.) and Meunier (J.), in Polarization, Matter and Radiation, published by Presses Universitaires, Paris, 1969.

[3] Meunier (J.), Cruchon (D.) and Bouchiat (M. A.), C. R. Acad. Sci., Paris, 1969, 268, 92.

[4] Bouchiat (M. A.) et Meunier (J.), J. Physique, 1971, 32, 561.

[5] Landau (L. D.) and Placzek (G.), Physik z. Sowjetunion, 1934, 5, 172.

[6] Mountain (R. D.), Rev. Mod. Phys., 1966, 38, 205.

[7] Meunier (J.), J. Physique, 1969, 30, 933.

[8] Montrose (C. J.), Solovyev (U. A.) and Litovitz (T. A.), J. Acoust. Soc. Am., 1968, 43, 117.
[9] Kestin (J.), Whitelaw (J. H.) and Zien (T. F.) Physica, 1964, 30, 161.

[10] Bouchiat (M. A.) and Langevin (D.), C. R. Acad. Sci., Paris, 1971, 272, 1357.

[11] Kadanoff (L. P.), and al., Rev. Mod. Phys., 1967, 39, 395, figure 10 .

[12] Sengers (J. V.) adn Keyes (P. H.), Phys. Rev. Letters, 1971, 26, 70 .

[13] White (J. A.) and Maccabee (B. S.), Phys. Rev. Letters, 1971, 26, 1468.

[14] Fisk (S.) and Wrdom (B.), J. Chem. Phys., 1969, 50, 3219.

[15] Cannell (D. S.) and Lunacek (J.), J. Physique, present issue. 